\title{
Conocimiento, acceso y uso del sistema de salud en adolescentes migrantes en Chile: resultados de un estudio exploratorio
}

\author{
Alexandra Obach', Felipe Hasen², Báltica Cabieses ${ }^{3}$, Cettina D 'Angelo², y Sylvia \\ Santander ${ }^{2}$
}

Forma de citar

Obach A, Hasen F, Cabieses B, D'Angelo C, Santander S. Conocimiento, acceso y uso del sistema de salud en adolescentes migrantes en Chile: resultados de un estudio exploratorio. Rev Panam Salud Publica. 2020;44:e175. https://doi.org/10.26633/ RPSP.2020.175

RESUMEN

Objetivo. Identificar el conocimiento, acceso y uso del sistema de salud por parte de adolescentes migrantes, y las barreras para el acceso y uso del sistema de salud en dicha población en Chile.

Métodos. Estudio exploratorio observacional, de corte transversal, aplicado en jornada participativa con adolescentes migrantes $(\mathrm{N}=30)$ de tres comunas de la ciudad de Santiago, Chile. Estudio multimétodos. Se aplicó una encuesta basal sobre el proceso migratorio y las experiencias en el sistema de salud, con análisis descriptivo de datos. Desde lo cualitativo, se realizaron grupos de discusión $(n=5)$ y talleres participativos $(n=2)$ con adolescentes migrantes.

Resultados. El 60\% de adolescentes afirmó desconocer si está inscrito en el sistema de salud, y la mitad no lo ha utilizado. Se identificó que el tiempo de residencia es un factor relevante para el uso efectivo de algunas prestaciones de salud. Se reconocieron barreras administrativas para acceder al sistema de salud, como situación migratoria, percepción de obligatoriedad de compañía de adultos, y experiencias de discriminación en la atención. Se identificaron necesidades específicas en salud sexual y reproductiva, como acceso a preservativos, y salud mental en relación a la atención de especialistas. Como facilitadores del acceso y uso se destacaron las experiencias de buen trato, y la presencia del sector salud en las escuelas.

Conclusiones. Se identificó desconocimiento del sistema de salud, y diversas barreras de acceso y uso de este por parte de adolescentes migrantes. En base a los resultados se proponen recomendaciones generales para el país y la región referidas a la promoción de la salud de este grupo.

Palabras clave Emigración e inmigración; adolescentes; sistemas de salud; América Latina; Chile.

En las últimas décadas, Chile ha enfrentado el desafío de constituirse en país receptor de población migrante internacional, proveniente principalmente de países de América Latina. A diciembre del 2019 se estimaban 1492522 migrantes internacionales en Chile, aproximadamente un $7 \%$ de la población total. Los principales países de origen eran Venezuela, Perú y Haití (1). Según el Censo 2017, del total de población adolescente del país (10 a 19 años) un 3\% correspondía a extranjeros (2). Este grupo

\footnotetext{
1 Universidad del Desarrollo, Santiago, Chile. $\bowtie$ Alexandra Obach, aobach@udd.cl

2 Ministerio de Salud de Chile, Santiago, Chile
}

etario es relativamente desconocido, al menos en términos de necesidades de vida y salud, así como en su consideración específica en investigación y políticas públicas $(3,4)$. Recién en la última década se ha puesto foco en el impacto de la migración en adolescentes, reconociéndolos como actores relevantes que, en muchas ocasiones, no son quienes toman la decisión de migrar (5).

Existe una serie de problemáticas en adolescentes migrantes que los exponen a riesgos en salud (4). Por ejemplo, suelen 
insertarse en el país receptor a través de las escuelas, donde se ha documentado situaciones de discriminación e inadaptabilidad $(6,7)$, así como experiencias traumáticas de adolescentes en zonas fronterizas (9). Junto con lo anterior, estudios documentan que los adolescentes pueden ser articuladores lingüísticos y culturales para sus familias y comunidades con la sociedad receptora, con posibles cargas asociadas a estas tareas (8).

En cuanto al acceso y el uso del sistema de salud, si bien en Chile se han desplegado importantes acciones para la población migrante internacional (10), aún se evidencia el incumplimiento del pleno derecho a la salud de esta población, y de niños, niñas y adolescentes migrantes en particular (11). Se identifican barreras de acceso y uso del sistema de salud hacia adolescentes migrantes, algunas de las cuales son estructurales y compartidas con la población nacional $(12,13)$, mientras que otras son específicas para este grupo. Entre ellas, barreras idiomáticas $(14,15)$, de acceso a salud mental (16-19), falta de documentación (20), de acceso a la información $(21,22)$, y de acceso a la salud sexual y reproductiva (SSR) (23-25).

El presente artículo describe los resultados del primer Encuentro participativo de adolescentes migrantes en salud, realizado el año 2019 por el Programa Nacional de Salud Integral de Adolescentes y Jóvenes del Ministerio de Salud de Chile (MINSAL) (26), cuyo objetivo fue identificar el conocimiento, acceso y uso del sistema de salud por parte de adolescentes migrantes, y las barreras para el acceso y uso del sistema de salud en dicha población en Chile.

\section{MÉTODOS}

Estudio exploratorio observacional, descriptivo y de corte transversal, en el marco del primer Encuentro participativo de adolescentes migrantes en salud, experiencia piloto de un día de duración realizada en el mes de agosto del 2019 en Santiago de Chile. Participaron 30 adolescentes migrantes, hombres y mujeres de diversas nacionalidades, con predominio de Venezuela, seguida por Colombia y la República Dominicana. Esta experiencia exploratoria es útil como línea de base para futuras intervenciones similares en Chile y la región.

Diseño multimétodos, con recogida simultánea de información cuantitativa y cualitativa $(27,28)$. Se realizó un muestreo opinático de acuerdo con criterios pragmáticos y de factibilidad (29). Se reclutó a adolescentes migrantes de entre $8^{\circ}$ básico y $3^{\circ}$ medio de tres escuelas públicas de las comunas de Independencia, Recoleta y Santiago, seleccionadas debido a su alta densidad de población migrante. El MINSAL contactó a las directivas de las escuelas, quienes en conjunto con docentes invitaron a adolescentes migrantes a participar. Criterios de inclusión: (i) estudiantes de las escuelas seleccionadas, (ii) entre $8^{\circ}$ básico y $3^{\circ}$ medio, y (iii) migrantes internacionales. Se estableció un cupo máximo de 30 participantes, 10 por escuela, con base en la viabilidad en términos de espacio físico, alimentación y traslado de los estudiantes, que estuvo a cargo del MINSAL. Cada escuela completó su cupo máximo, excluyendo con ello a estudiantes interesados en participar pero que quedaron fuera por no haber más cupos disponibles.

\section{Metodología cuantitativa}

Se aplicó una encuesta transversal, de diagnóstico basal, que incluía preguntas sobre: (i) datos sociodemográficos, (ii) antecedentes del proceso migratorio y, (iii) experiencia con el sistema de salud. El Cuadro 1 da cuenta de las variables de estudio. La información fue tabulada en una base de datos Excel (Microsoft

CUADRO 1. Variables de encuesta breve a adolescentes migrantes internacionales en Chile

\begin{tabular}{|c|c|c|}
\hline Dimensión & Variable & Tipo de variable \\
\hline \multirow[t]{4}{*}{ Datos sociodemográficos } & Género & Multinomial (femenino/masculino/otro) \\
\hline & Edad & Continua (en años cumplidos) \\
\hline & Número de miembros del núcleo familiar en Chile con quienes vive & Ordinal (de conteo, número de personas) \\
\hline & Con quiénes vive en Chile & Multinomial: solo, padres, familiares, amigos, pareja, hijos \\
\hline \multirow[t]{3}{*}{ Antecedentes del proceso migratorio } & Tiempo de residencia en Chile & Ordinal (de conteo, número de personas) en años \\
\hline & De quién fue la decisión de migrar & Multinomial: mía, de mis padres, otros familiares \\
\hline & Con quiénes migraron & Multinomial: solo, padres, familiares, amigos, pareja, hijos \\
\hline \multirow{13}{*}{ Experiencia con el sistema de salud } & Accedió alguna vez al sistema de salud chileno & Binaria (sí/no) \\
\hline & Tipo de previsión de salud & Multinomial: pública, privada, otra, no sé, ninguna \\
\hline & $\begin{array}{l}\text { Conocimiento sobre el Programa Espacios Amigables para la } \\
\text { salud de adolescentes en centros de salud }\end{array}$ & Binaria (sí/no) \\
\hline & Sabe dónde debe acudir en caso de necesitar atención psicológica & Binaria (sí/no) \\
\hline & $\begin{array}{l}\text { Sabe a qué consultorio u hospital debe acudir para una consulta } \\
\text { médica }\end{array}$ & Binaria (sí/no) \\
\hline & Sabe dónde acudir para atención dental & Binaria (sí/no) \\
\hline & Sabe dónde ir en caso de necesitar algún método anticonceptivo & Binaria (sí/no) \\
\hline & Uso alguna vez de espacios amigables & Binaria (sí/no) \\
\hline & Uso alguna vez de atención médica & Binaria (sí/no) \\
\hline & Uso alguna vez de atención dental & Binaria (sí/no) \\
\hline & Percepción de discriminación en Chile & Binaria (sí/no) \\
\hline & Lugares donde se ha sentido discriminado & Multinomial: lugares públicos, colegios, centros de salud, otros \\
\hline & Razón de haberse sentido discriminado & Multinomial: género, edad, nacionalidad, color de piel, barrio, otro \\
\hline
\end{tabular}

Fuente: Elaboración propia, basado en el estudio original Reporte de Sistematización 1er Encuentro participativo de adolescentes y jóvenes migrantes en salud. Santiago: MINSAL; 2019. 
Corp.) y se utilizó el programa IBM SPSS (IBM Corp.) para el análisis descriptivo y bivariado. Respecto del análisis descriptivo, para las variables continuas se obtuvieron medias y desviación estándar, y para las categóricas frecuencias absolutas y porcentajes. Para el análisis bivariado se realizó prueba de comparación de proporciones $\mathrm{Chi}^{2}$ para comparar diferencias según el tiempo de residencia en Chile. Para variables con menos de 5 respuestas por celdilla, se aplicó el test exacto de Fisher.

\section{Metodología cualitativa}

Se consideró un diseño de estudio de caso (30), con: (i) grupos de discusión ( $n=5$, de 6 participantes por grupo); (ii) talleres plenarios participativos $(n=2$, cada uno de ellos con el total de participantes de la jornada). Los grupos de discusión contaron con un guión de entrevista semiestructurado referido a: (i) conocimiento, acceso y uso del sistema de salud (con preguntas en torno a conocimiento e inscripción al sistema de salud chileno); y (ii) barreras para el acceso y uso, y experiencia con el sistema de salud y con el Centro de Salud.

En el Taller 1 se presentó un video sobre barreras que enfrentan los adolescentes en salud, y luego se les pidió que identificaran sus necesidades en salud y las barreras vividas de acceso y uso del sistema de salud en Chile. En el Taller 2 se realizó un ejercicio de imaginería para que identificaran sus demandas hacia al sistema de salud. Para ambos talleres, cada participante depositó sus respuestas individuales anónimas en cajas dispuestas para este fin por el equipo de investigación. Los audios de las entrevistas y las notas de campo fueron transcritas en forma literal. Todo el material cualitativo fue analizado temáticamente (31) con apoyo del programa informático NVivo (QSR International).

El proyecto contó con la aprobación del MINSAL y su fase de estudio fue aprobada por una Comisión Técnica Ético-Social de Migración y Salud del MINSAL (Res. Ex. N 42 del 12 de agosto 2019). Todos los participantes mayores de edad firmaron un consentimiento informado, y los menores de edad (17 años y menos) un asentimiento informado y el consentimiento de padres o cuidadores antes de su participación. La encuesta fue anónima y las transcripciones de grupos de discusión y plenarias contaron con códigos confidenciales. Para los grupos de discusión se utilizó la letra $\mathrm{G}$ y un número para designar el grupo correspondiente, y H/M para identificar el sexo del o de la participante. Dado que en los talleres participativos las respuestas fueron anónimas, no se les asignó códigos.

\section{RESULTADOS}

\section{Resultados cuantitativos}

El cuadro 2 detalla la información sociodemográfica de los participantes. El rango etario de la población estudiada fue de 12-28 años, y el promedio de edad fue de 15,23 años (DE 2,9). De los 30 participantes, el promedio de estadía en Chile fue de 34,4 meses (DE 16,4). Del total, 8 reportaron llevar menos de dos años en Chile, 16 reportaron llevar dos años y más, y 6 no respondieron.

La mayoría de los participantes vive con alguno de sus padres (90\%) y, en algunos casos, conviven también con otros familiares. La decisión de migrar a Chile fue principalmente de los padres, madres o tutores (76\%). En ningún caso viajó el grupo familiar completo. La mitad de los encuestados dice no haber utilizado el sistema de salud en Chile. Junto con ello, el $60 \%$ no sabe si está inscrito formalmente en el sistema de salud, y un $25 \%$ indicó no estar inscrito.

El 60\% no conoce el "Programa Espacios Amigables para la salud de adolescentes en centros de salud" (en adelante, Espacios Amigables) que se ha instalado progresivamente en Chile con el fin de contribuir al bienestar y resolver necesidades de salud de adolescentes entre 10 y 19 años. El 56\% no sabe dónde debe acudir en caso de necesitar atención psicológica y el $68 \%$ de los adolescentes migrantes sabe a qué consultorio u hospital debe acudir para una consulta médica. Si bien el 60\% dice saber a qué servicio del sistema de salud acudir en caso de necesitar algún método anticonceptivo, el 76\% no ha acudido ni solicitado métodos anticonceptivos. El 52\% dice haber recibido atención dental, el $44 \%$ acudió a consulta médica y solo el 32\%

CUADRO 2. Información sociodemográfica de los participantes $(n=30)$

Características sociodemográficas $\%$

Género

Femenino N

Masculino 43,33

Otro

Sin respuesta

Total

50

3,33

3,33

100

\section{Año escolar en curso}

Séptimo y octavo básico

Enseñanza media $\left(1^{\circ}-3^{\circ}\right.$ medio)

Total

53,32

46,66

100

\section{Zona de la Región Metropolitana en que vive}

Norte

63,32

Centro

23,33

Sur Oriente

6,66

No especificada

6,66

Total

100

\section{Comuna del establecimiento educacional}

Norte (comunas de Recoleta e Independencia)

Centro (comuna de Santiago)

Sin respuesta

Total

\section{2}

30

País de nacimiento

Bolivia

Chile

Colombia

Ecuador

Haití

Perú

República Dominicana

Venezuela

Total

71,43

28,57

6,66

100

Fuente: Elaboración propia, basada en datos recogidos durante el Encuentro participativo de adolescentes migrantes en salud de 2019 desarrollado por el MINSAL. 
concurrió alguna vez a un Espacio Amigable. Un 12\% indica haberse sentido discriminado alguna vez en Chile en lugares públicos, colegios y centros de salud. La principal razón de discriminación fue su nacionalidad, seguido por el color de piel.

Cuando se compara el uso de servicios de salud $(n=24)$ según el tiempo de estadía en Chile (menos de dos años versus dos o más años en Chile) (Cuadro 3), se observa que solo dos prestaciones mostraron diferencias significativas. La primera es el haber tenido alguna atención médica, siendo más alto en quienes llevaban dos años o más en Chile comparado con menos de dos años (10 versus 4 participantes, $\mathrm{p}=0,04$ ). La segunda es si alguna vez usó Espacios Amigables, también más alto en quienes llevaban dos o más años $(p=0,03)$. Las demás categorías no mostraron diferencias estadísticas según el tiempo de residencia en Chile.

\section{Resultados cualitativos}

Conocimiento del sistema de salud. La mayoría de los participantes manifestó tener escasa o nula información del sistema de salud chileno. Quienes están inscritos, reconocen no saber mucho de este o no haberlo utilizado nunca. Sostienen falta de claridad respecto del proceso de inscripción y desinformación sobre si se requiere la compañía de un adulto:

"Yo no sé cómo funciona"(G1H).

"No sé si estoy inscrito"(G2H).

"Sí, también creo que falta más información. Que se asegure bien qué papeles son necesarios para inscribirse"(G2M).

Los resultados del Taller 1 son coherentes con la información proveniente de los grupos de discusión, y refuerzan la idea de falta de información en relación con el sistema de salud.

CUADRO 3. Acceso y uso de servicios de salud ( $n=24$ participantes) según el tiempo de estadía en Chile

\begin{tabular}{|c|c|c|c|}
\hline Tipo de servicio & $\begin{array}{l}\text { Menos de dos } \\
\text { años en Chile } c \\
\quad(\mathrm{n}=8)\end{array}$ & $\begin{array}{l}\text { Dos o más } \\
\text { años en Chile } \\
\quad(n=16)\end{array}$ & $\begin{array}{l}\text { Valor p (Chi2 } \\
\text { Fisher) }\end{array}$ \\
\hline $\begin{array}{l}\text { Alguna vez atención de salud } \\
\text { Sí } \\
\text { No }\end{array}$ & $\begin{array}{l}4 \\
4\end{array}$ & $\begin{array}{l}8 \\
8\end{array}$ & 0,052 \\
\hline $\begin{array}{l}\text { Alguna vez atención médica } \\
\text { Sí } \\
\text { No }\end{array}$ & $\begin{array}{l}4 \\
4\end{array}$ & $\begin{array}{c}10 \\
6\end{array}$ & $0,048^{*}$ \\
\hline $\begin{array}{l}\text { Alguna vez atención de emergencia } \\
\text { Sí } \\
\text { No }\end{array}$ & $\begin{array}{l}2 \\
6\end{array}$ & $\begin{array}{c}6 \\
10\end{array}$ & 0,098 \\
\hline $\begin{array}{l}\text { Alguna vez usó espacios amigables } \\
\text { Sí } \\
\text { No }\end{array}$ & $\begin{array}{l}1 \\
7\end{array}$ & $\begin{array}{l}9 \\
7\end{array}$ & $0,031^{*}$ \\
\hline $\begin{array}{l}\text { Alguna vez recibió MAC } \\
\text { Sí } \\
\text { No }\end{array}$ & $\begin{array}{l}1 \\
7\end{array}$ & $\begin{array}{c}5 \\
11\end{array}$ & 0,061 \\
\hline $\begin{array}{l}\text { Alguna vez recibió atención psicológica } \\
\text { Sí } \\
\text { No }\end{array}$ & $\begin{array}{l}2 \\
6\end{array}$ & $\begin{array}{c}5 \\
11\end{array}$ & 0,059 \\
\hline $\begin{array}{l}\text { Alguna vez recibió atención dental } \\
\text { Sí } \\
\text { No }\end{array}$ & $\begin{array}{l}4 \\
4\end{array}$ & $\begin{array}{l}9 \\
7\end{array}$ & 0,092 \\
\hline
\end{tabular}

Fuente: Elaboración propia, basada en datos recogidos durante el Encuentro participativo de adolescentes migrantes en salud de 2019, desarrollado por el MINSAL.
Barreas de acceso y uso del sistema de salud. La principal barrera de acceso es administrativa, para la inscripción en el sistema de salud, vinculada a la regularización migratoria de ellos y sus padres/cuidadores.

"Yo duré casi un año sin atenderme por la falta de los papeles" (G1M).

Quienes han accedido al sistema de salud lo perciben positivo en términos técnicos, pero frío y distante $y$, en algunos casos, discriminatorio.

"Me parece excelente, excepto en la atención. Porque cuando uno llega y va a decir los síntomas, ni te ven a la cara ni te prestan atención, y es como que todo lo que dices va directo a la computadora y no hay un diálogo. Y no prestan atención a lo que tienes, no te sientes atendido. Eso es lo primero que debería cambiar" (G3H).

Respecto de la atención hospitalaria, sorprenden las listas de espera y el hecho de ser tratados como adultos. Una demanda hacia el sistema de salud es que su especificidad como adolescentes sea considerada en la atención.

"O sea, como que deben darles una prioridad a los adolescentes, porque igualmente están saliendo de la infancia y entrar a la adolescencia. Como que deberían entenderlo más" (G1M).

"Creo que necesitamos también que nos tomen en cuenta, porque muchas veces ser adolescente es difícil, porque estás en la etapa en que no eres ni niño ni adulto..." (G5H).

La información recogida en el Taller 1 confirma las barreras expresadas mediante los grupos de discusión, agregando elementos como la carencia de previsión de salud y el costo de la atención.

Barreras de acceso a la salud sexual y reproductiva (SSR). Una de las prioridades identificadas es el acceso a preservativos. Los participantes sostuvieron que en las farmacias los preservativos son muy caros y les avergüenza comprarlos:

"Cuando uno va a la farmacia a comprar condones, el señor va a pensar que uno va a eso, entonces no debe importar lo que piensen, pero igual importa, da vergüenza ir y preguntar por los condones, cuáles son los más económicos, los más buenos, cualquier cosa" (G4H).

Respecto de la obtención de preservativos en la red de atención primaria, manifiestaron que solo pueden ser solicitados por adultos, aun cuando la Ley $\mathrm{N}^{\circ} 20418$ establece lo contrario. Sin embargo, perciben como algo positivo tener acceso a matronas en las escuelas, ya que así pueden acceder a los preservativos de manera directa y resguardando su privacidad. Algunos de los adolescentes entrevistados comparan la situación de esta temática en sus países de origen y la experimentada en Chile:

"[En mi país] Me los regalaban, pero acá es superdistinto, tiene que pedir hora, la matrona te da una receta para que pueda retirarlo. Mucho trámite. Entonces, si uno tiene una urgencia y no tiene dinero para comprar condones en una farmacia, igual es como un riesgo" (G5H). 
"Mi mamá me los pide en el consultorio. Me dijo que se los dan a un mayor, por eso los recibe ella. Yo tengo 15 años" (G5M).

"En el colegio hay una matrona y me queda más cerca que ir al doctor" (G1H).

En el Taller 2, se pudo identificar como necesidades en salud por parte de los participantes el acceso a la educación sexual y, tal como se menciona en los grupos de discusión, el acceso a matronas en las escuelas.

Barreras de acceso y adherencia a salud mental. Los entrevistados percibieron que las diferencias culturales, el desconocimiento del territorio local, las experiencias de discriminación y el estar lejos de sus seres queridos afecta su salud mental:

"A mí me pasa que los amigos de ahora no significan tanto como los de allá [país de origen]; me siento solo" (G5H).

"Nosotros los caribeños vivimos muy en comunidad; eso es una de las cosas por las que también muchos estudiantes entran en depresión por eso" (G3M).

Respecto al suicidio o la ideación suicida en adolescentes migrantes, algunos lo asocian a una crisis identitaria vinculada al proceso migratorio:

"Y de hecho se suicidó un niño la semana pasada" (G2M).

En vista de lo anterior, preocupan las barreras percibidas por los adolescentes relativas a la atención y derivación oportuna con especialistas de salud mental. Quienes han accedido a esta atención de especialidad relatan baja adherencia debido a experiencias negativas:

"En realidad no sirve, porque uno va a una consulta y [no] le dice ni la mitad, solo el principio de lo que te está pasando, y luego te vuelve a atender después de un mes y la conversación se queda en el aire (...); la consulta debería ser más larga y seguida, yo pues salgo igual, o a veces hasta peor" (G1M).

Los resultados del Taller 2 enfatizan también la necesidad de información respecto al consumo de drogas.

Aspectos facilitadores en el acceso a salud. El buen trato recibido por parte de trabajadores de la salud se reconoció como un poderoso motivador para acceder al sistema. Al respecto, quienes han utilizado los Espacios Amigables tienen una buena percepción de esta estrategia, que permite la atención en salud sin la presencia de un adulto y con mayor confidencialidad y cercanía. Valoran también la existencia de Espacios Amigables al interior de las escuelas porque permite iniciar métodos de anticoncepción, acceder a preservativos y a información en SSR y salud mental.

"Bueno, yo tuve la experiencia en el Espacio Amigable del liceo, fue superchévere. Es una oportunidad buena; iban muchas personas" (G3M).

\section{DISCUSIÓN}

Este estudio buscó identificar el conocimiento, acceso y uso del sistema de salud por parte de adolescentes migrantes, y las barreras para el acceso y uso del sistema de salud en dicha población. Los resultados dan cuenta de un desconocimiento generalizado por parte de los adolescentes migrantes respecto del sistema de salud en Chile. Como principales barreras de acceso y uso del sistema, destacan la percepción de necesitar contar con papeles migratorios regularizados, la compañía de un adulto como requisito para la atención de salud, y las experiencias de discriminación sufridas en el sistema de salud. También surgieron barreras específicas en SSR (referidas al acceso a preservativos y anticonceptivos) y en salud mental. Destacan como facilitadores algunas experiencias de buen trato, y la presencia de personal de salud en las escuelas.

Los adolescentes migrantes viven procesos de transnacionalización a edades tempranas, experimentando diversas potenciales vulnerabilidades que los exponen a riesgos en salud en distintos ámbitos. Por esto, se hace urgente identificar el conocimiento, acceso y uso del sistema de salud por parte de adolescentes migrantes, y las barreras que enfrentan en Chile y la región. Solo comprendiendo esta realidad se podrán generar acciones desde los Estados y la sociedad civil que apunten a una mayor equidad en materia de salud de los adolescentes migrantes.

Los resultados del estudio son coherentes con lo que evidencia la literatura científica en migración y salud, dando cuenta de barreras similares en el acceso y uso del sistema de salud por parte de la población migrante internacional adolescente (32) y adulta (33). Asimismo, este estudio sugiere que los adolescentes migrantes deben sobrellevar barreras similares a la población adolescente nacional $(12,13,34,35)$, además de algunas barreras adicionales como desconocimiento, idioma y no contar con previsión formal de salud. El tiempo de residencia es un factor relevante para el uso efectivo de algunas prestaciones de salud en este grupo, lo que coincide con la literatura internacional en esta población y en migrantes adultos $(21,23)$. Se relatan experiencias de discriminación en el sistema de salud, necesidades en salud mental, y la oportunidad de integrar servicios de salud para adolescentes en las escuelas.

Este artículo da cuenta de los resultados de una iniciativa piloto diagnóstica y exploratoria de la situación de adolescentes migrantes con respecto al sistema de salud en Chile. El estudio fue desarrollado durante un taller inédito liderado por el Programa Nacional de Salud Integral de Adolescentes y Jóvenes del MINSAL, junto a un equipo de investigación dedicado a la temática. Sus resultados pueden ser de interés para trabajadores de la salud y organizaciones de migrantes o gubernamentales de diversos países de América Latina, pues identifica problemáticas que son globales, como las oportunidades de acceso y las barreras de uso efectivo de los servicios de salud para estas poblaciones. A partir de sus resultados, se proponen recomendaciones para ser adaptadas a diversos contextos, entendiendo que los adolescentes migrantes requieren desde distintas instancias formales ser reconocidos, valorados e integrados en los sistemas de salud.

Se reconocen algunas limitaciones del estudio. Primero, la selección por conveniencia de escuelas públicas de la Región Metropolitana, lo que dejó fuera a escuelas subvencionadas y privadas que pueden tener otras experiencias migratorias y de SSR. Segundo, la selección de los participantes a través de los directivos de cada escuela podría haber limitado la participación de adolescentes migrantes con características diversas como otro idioma nativo o alguna otra consideración académica o personal no reconocida. Esto induce a posibles riesgos de sesgo de selección, al haberse potencialmente 
excluido a adolescentes con distintas experiencias migratorias y de salud. Tercero, el tamaño acotado de la muestra limita interpretaciones de patrones generales de esta población adolescente migrante en Chile y la región. Cuarto, los estudiantes estuvieron la mayor parte del tiempo en presencia de sus profesores, lo que podría haber influenciado algunas respuestas. Quinto, la actividad tuvo un solo día de duración y el equipo que facilitaba el taller tuvo poco tiempo para desarrollar un ambiente de confianza para el diálogo, lo que también podría haber limitado la expresión de algunas respuestas. Como fortaleza se reconoce que es el primer estudio de su tipo en Chile, organizado de manera conjunta entre la academia y la autoridad sanitaria. Permitió generar información relevante e inédita sobre salud en adolescentes migrantes en Chile, para la construcción y adecuaciones sanitarias y de políticas de salud.

\section{Conclusiones y recomendaciones}

Se identificó un desconocimiento respecto del sistema de salud en general, así como sobre el acceso y uso de este por parte de los adolescentes migrantes. Como principales barreras para el acceso y uso del sistema de salud en dicha población destacaron las barreras administrativas referidas a su condición migratoria, la percepción por parte de los adolescentes que deben estar acompañados por un adulto, y barreras específicas en SSR referidas al acceso a preservativos, y a salud mental en relación con el acceso a especialistas en esta área. Destacaron experiencias de buen trato por parte de trabajadores de la salud, y la presencia del sector salud en las escuelas en tanto facilitadores para el acceso y uso del sistema de salud por parte de los adolescentes migrantes.

A partir de estos resultados, se proponen recomendaciones generales para el país y la región: a) generar o fortalecer mecanismos de información sobre el sistema de salud para migrantes adolescentes; b) realizar o fortalecer acciones de acercamiento al sistema de salud en las escuelas, sin olvidar a los adolescentes migrantes que están fuera del sistema educativo; c) fortalecer el buen trato a adolescentes migrantes, rechazando toda forma de discriminación, desde un enfoque de interculturalidad en salud; d) fortalecer el trabajo intersectorial entre salud y educación, orientado a mejorar el acceso a preservativos y métodos de anticoncepción en las escuelas a través de estrategias como los Espacios Amigables; e) fortalecer la promoción y prevención en salud mental en las escuelas, facilitando los mecanismos de derivación de adolescentes migrantes a especialistas.

Contribución de los autores. AO, FH, BC, CD participaron en el diseño del estudio original, la recopilación, análisis e interpretación de datos, así como en la redacción, revisión y corrección del manuscrito final para su aprobación. SS participó en la redacción, revisión y corrección del manuscrito final. Todos los autores aprobaron la versión final.

\section{Financiamiento. Ministerio de Salud de Chile.}

Agradecimientos. A todos los adolescentes que participaron en el primer Encuentro participativo de adolescentes migrantes en salud, llevado a cabo en Santiago de Chile en 2019.

\section{Conflicto de interés. Ninguno declarado.}

Declaración. Las opiniones expresadas en este manuscrito son únicamente responsabilidad de los autores y no reflejan necesariamente los de la Revista Panamericana de Salud Pública o la Organización Panamericana de la Salud.

\section{REFERENCIAS}

1. Instituto Nacional de Estadísticas de Chile. Estimación de personas extranjeras residentes habituales en Chile al 31 de diciembre 2019. Informe Técnico. Instituto Nacional de Estadísticas, Departamento de Extranjería y Migración. Gobierno de Chile; 2020.

2. Encuesta de Caracterización Socioeconómica (CASEN). Santiago: Ministerio de Desarrollo Social, Gobierno de Chile; 2017.

3. Reyes Eguren A. Juventudes migrantes. Indocumentados, invisibilizados y mitificados. Marco conceptual para una agenda de investigación en el estudio de la migración juvenil. Revista de El Colegio de San Luís, Nueva Época. 2013;3(5): 287-307.

4. Stefoni C. La construcción del campo de estudio de las migraciones en Chile: notas de un ejercicio reflexivo y autocrítico. Iconos. Revista de Ciencias Sociales. 2017; 58: 109-129. doi: http://dx.doi. org/10.17141/iconos.58.2017.2477

5. Porraz Gómez I. Juventud migrante del sur. Apuntes para su construcción conceptual. Revista Pueblos y Fronteras. 2015;10(20): 171-194.

6. Salas N, Castillo D, San Martín C, Kong, Thayer L, Huepe D. Inmigración en la escuela: caracterización del prejuicio hacia escolares migrantes en Chile. Universitas Psychologica. 2017;16(5): 1-15.

7. Stefoni C, Acosta E, Gaymer M, Casas-Cordero F. Niños y niñas inmigrantes en Santiago de Chile. Entre la integración y la exclusión. Santiago: Universidad Alberto Hurtado/OIM; 2008.

8. Akiyama T, Win T, Maung C, Ray P, Sakisaka K, Tanabe A, et al. Mental health status among Burmese adolescent students living in boarding houses in Thailand: a cross-sectional study. BMC Public Health. 2013;13:337.
9. Santos M, Gorunkanti A, Jurkunas L, Handley M. 2018. The Health Literacy of U.S. Immigrant Adolescents: A Neglected Research Priority in a Changing World. Int J Environ Res Public Health. 2018;15:3-18. doi:10.3390/ijerph15102108.

10. Ministerio de Salud de Chile. Política de salud de migrantes internacionales. Santiago: Ministerio de Salud de Chile; 2017.[0]

11. Bernales M, Cabieses B, McIntyre AM, Chepo M, Flaño J, Obach A. Determinantes sociales de la salud de niños migrantes internacionales en Chile: evidencia cualitativa. Salud pública de México. 2018;60(5):566-78.

12. Sadler M, Obach A, Luego, X, Biggs A. Estudio Barreras de acceso a los servicios de salud para la prevención del embarazo adolescente en Chile. Santiago: Ministerio de Salud; 2010.

13. Ramírez GR, Bravo PE, Vivaldi MIM, Manríquez IP, Pérez TG. Acceso a anticoncepción en adolescentes: Percepciones de trabajadores de la salud en Huechuraba, Chile. Rev Panam Salud Publica. 2017;41:1-8.

14. Banas JR, Ball JW, Wallis LC, Gershon S. The Adolescent Health Care Broker-Adolescents Interpreting for Family Members and Themselves in Health Care. J Community Health. 2017;42(4):739-47.

15. Lee SK, Sulaiman-Hill CR, Thompson SC. Overcoming language barriers in community-based research with refugee and migrant populations: Options for using bilingual workers. BMC Int Health Hum Rights. 2014;14(1):1-13.

16. Saunders NR, Gill PJ, Holder L, Vigod S, Kurdyak P, Gandhi S, et al. Use of the emergency department as a first point of contact for 
mental health care by immigrant youth in Canada: A population-based study. CMAJ. 2018;190(40):E1183-91.

17. Islam F, Multani A, Hynie M, Shakya Y, McKenzie K. Mental health of South Asian youth in Peel Region, Toronto, Canada: A qualitative study of determinants, coping strategies and service access. BMJ Open. 2017;7(11):1-11.

18. Posselt M, McDonald K, Procter N, De Crespigny C, Galletly C. Improving the provision of services to young people from refugee backgrounds with comorbid mental health and substance use problems: Addressing the barriers. BMC Public Health. 2017; 17(1):1-17.

19. McCann TV, Mugavin J, Renzaho A, Lubman DI. Sub-Saharan African migrant youths' help-seeking barriers and facilitators for mental health and substance use problems: A qualitative study. BMC Psychiatry [Internet]. 2016;16(1):1-10. Disponible en: http:// dx.doi.org/10.1186/s12888-016-0984-5

20. Myhrvold T, Småstuen MC. The mental healthcare needs of undocumented migrants: an exploratory analysis of psychological distress and living conditions among undocumented migrants in Norway. J Clin Nurs. 2017;26(5-6):825-39.

21. Straiton ML, Myhre S. Learning to navigate the healthcare system in a new country: a qualitative study. Scand J Prim Health Care [Internet]. 2017;35(4):352-9. Disponible en: https://doi.org/10.1080/028 13432.2017.1397320

22. Kim W, Kreps GL, Shin CN. The role of social support and social networks in health information-seeking behavior among Korean Americans: A qualitative study. Int J Equity Health [Internet]. 2015;14(1):1-10.

23. Espinoza R, Martínez I, Levin M, Rodriguez A, Chan T, Goldenberg $S$, et al. Cultural perceptions and negotiations surrounding sexual and reproductive health among migrant and non-migrant indigenous Mexican women from Yucatán, Mexico. J Immigr Minor Heal. 2014;16(3):356-64.

24. Reyes-Urueña J, Campbell C, Hernando C, Vives N, Folch C, Ferrer $\mathrm{L}$, et al. Differences between migrants and Spanish-born population through the HIV care cascade, Catalonia: An analysis using multiple data sources. Epidemiol Infect. 2017;145(8):1670-81.

25. Khuat T, Do T, Nguyen V, Vu X, Nguyen P, Tran K, et al. The Dark Side of Female HIV Patient Care: Sexual and Reproductive
Health Risks in Pre- and Post-Clinical Treatments. J Clin Med. 2018;7(11):402.

26. Ministerio de Salud. Sistematización 1er encuentro participativo de adolescentes y jóvenes migrantes en salud. Santiago: Programa Nacional Salud Integral de Adolescentes y Jóvenes; 2019.

27. Hernández R, Fernández C, Baptista P. Metodología de la investigación. $6^{a}$ ed. México: Mcgraw-Hill/Interamericana; 2014.

28. Creswell J. Research design: qualitative, quantitative, and mixed methods approaches. California: SAGE; 2014.

29. Vázquez ML, Ferreira M, Mogollón A, Frenández MJ, Delgado ME, Vargas I. Introducción a las técnicas cualitativas de investigación aplicadas en salud. Barcelona: Universidad Autónoma de Barcelona; 2005.

30. Creswell J. Qualitative inquiry \& research design. Choosing among five approaches. Sage Publications; 2013.

31. Thomas DR. A general inductive approach for qualitative data analysis. American Journal of Evaluation. 2006;27(2): 237-246.

32. Obach A, Cabieses B, Carreño A. Identificando a los adolescentes y jóvenes migrantes para su pertinente abordaje en salud. Cuad Méd Soc. 2018, 58(4):41-49.

33. Cabieses B, Oyarte M. Acceso a salud en inmigrantes: identificando brechas para la protección social en salud. Rev Saúde Pública. 2020;54:20. Disponible en: https://www.scielo.br/pdf/rsp/v54/15 18-8787-rsp-54-20.pdf

34. Obach A, Sadler M, Aguayo F, Bernales M. Salud sexual y reproductiva de hombres jóvenes en Chile: resultados de un estudio cualitativo Rev Panam Salud Publica. 2018;42:e124. https://doi. org/10.26633/RPSP.2018.124.

35. Obach A, Sadler M, Jofré N. Salud sexual y reproductiva de adolescentes en Chile: el rol de la educación sexual. Rev Salud Pública. 2017;19 (6):852-8.

Manuscrito recibido el 17 de julio de 2020; aceptado para publicación, tras revisión, el 20 de noviembre de 2020.

\section{Knowledge, access and use of the health system by migrant adolescents in Chile: results of an exploratory study}

ABSTRACT Objective. To identify the knowledge, access, and use of the health system by migrant adolescents, and the barriers to access and use of the health system in that population in Chile.

Methods. Observational, cross-sectional exploratory study applied in a participatory workshop with migrant adolescents $(\mathrm{N}=30)$ in three municipalities of the city of Santiago, Chile. Multimethods study. A baseline survey on the migration process and experiences in the health system was applied, with descriptive data analysis. From the qualitative point of view, discussion groups $(n=5)$ and participatory workshops $(n=2)$ were carried out with migrant adolescents.

Results. Sixty percent of the adolescents said they did not know if they were registered in the health system, and half of them had not used it. Time of residence was identified as a relevant factor for the effective use of some health benefits. Administrative barriers to access the health system were recognized, such as immigration status, perception of obligatory adult companionship, and experiences of discrimination in care. Specific needs were identified in sexual and reproductive health, such as access to condoms, and mental health care from specialists. As facilitators of access and use, experiences of proper treatment and the presence of the health sector in schools were highlighted.

Conclusions. Lack of information about the health system and various barriers to access and use by migrant adolescents were identified. Based on the results, general recommendations are proposed for the country and the region to improve the health of migrant adolescents.

Keywords $\quad$ Emigration and immigration; adolescent; health systems; Latin America; Chile. 